\title{
Intelligent Conversational Agent for Enhancement of Online Communication in Universities: An Overview of Kenyatta University
}

\author{
Isaac Kuria \& Harrison Njoroge \\ Kenyatta University, Nairobi, KENYA \\ Computing and Information Technology Department
}

Received: 18 November 2021 • Accepted: 24 December 2021 • Published Online: 30 December 2021

\section{Abstract}

University websites and online portals are the primary means through which potential students and other stakeholders find important information about an institution. University websites are essential to these organizations' marketing and communication efforts. In this paper, focus has been put on the need to complement these websites with the use of an AI Chatbot (UniBot) in order to serve more efficiently. This study aims at performing an extensive literature survey on intelligent conversational agents and the feasibility of applying them in enhancing online communication in universities. The study utilizes an iterative - incremental methodology to aid in design and development of UniBot, using AIML (Artificial Intelligent Markup Language) Pattern matching algorithm on the Pandorabot (AIAAS) platform, to generate high quality training data, with which, the agents Natural Language Understanding (NLU) model is trained. The study also provides for training and testing the agent using data which is acquired from Online Communication, University Website department at Kenyatta University.

Keywords: artificial intelligent markup language, AI Chatbot, natural language understanding.

\section{Introduction}

According to Melisa Perich (2015), a lot of research work has been done on Artificially Intelligent Systems to support education. Elis, Allen and Tuson (2010) argue that with the development of Artificial Intelligence, computer systems can complete or perform tasks that would require human intelligence at a much larger scale than we could on our own. AI has been applied in fields like speech recognition, visual perception, natural language processing and decision making. Artificially Intelligent Conversational Agents or Chatbots have being utilized in a number of ways. Companies like Paypal, Facebook and Tesla are increasingly relying on Artificially Intelligent Bots. Google has also developed Google Assistant which is a virtual agent that can engage users in two-way conversations. From these advancements we can clearly see that there is a growth in Artificially Intelligent Conversational Agents or Chatbots adoption rate. Modern commercial chatbots offer more advanced development environments enabling the design and development of intelligent conversational agents with complex and goal driven behavior.

(C) Authors. Terms and conditions of Creative Commons Attribution 4.0 International (CC BY 4.0) apply. Correspondence: Harrison Njoroge, Kenyatta University, Computing and Information Technology Department, Nairobi, KENYA. E-mail: njoroge.harrision@ku.ac.ke. 


\section{Literature review}

The development of conversational agents enabled human beings to interact with computers by use of natural language processing (NLP). According to Atwell and Shawar, (2007), with the growth in pervasive computing and technologies, there developed an ardent requirement to interact with computers in the same way communication is done with people, by using natural language. According to (Zadrozny et al. 2000), by allowing users to express themselves directly and in a natural way, i.e., by speaking, typing, and pointing, a more sophisticated HCI can be achieved. Milan Van Eeuwen (2016) defines a chatbot as an overall term for describing concepts like chatter bots, virtual agents, and conversational agents. Desaulniers (2016) defined a chatbots as an interactive messaging application powered by artificial intelligence (AI). In addition to Desaulniers definition, Schlicht (2016) describes a chatbot as a service, powered by rules and sometimes artificial intelligence that you interact with using a chat interface. A chatbot is defined in various ways but they all describe the same phenomenon. The definitions mostly differ in mentioning to what extent a chatbot is driven by artificial intelligence. With more progress in research, Milan van Eeuwen (2017) describes a chatbot as an intelligent application that interacts with user in natural language through a chat and can be used effectively to serve a commercial purpose. According to microsoft.com (2018), Microsoft Cortana has some services like weather forecast, calendars, reading outlook emails, giving time estimations for travel, giving directions, and integrating with OneNote to show users' their notes. According to Google Assistant (2018), Google Assistant is an extension of the basic OK Google functionality that allows users to conduct search and control their mobile devices through voice commands. Assistant is programmed into Google phones, Android OS and will be integrated into some cars. According to amazon.com (2018) (retrieved from https://developer.amazon.com/alexa), Alexa can access the weather, connect to radio and television stations, and has partnerships with a number of services, including: JustEat, Uber, FitBit, The Telegraph, Spotify and Nest, among others. These services can be accessed with the Alexa interface.

\section{Evaluation and results}

\subsection{Approach}

Black-box approach which is qualitative assessment was chosen to evaluate UniBot. Black-box approach focuses on the output generated in response to the given input without concern with the internal processing. This approach suits the nature of UniBot well because the core function of UniBot is to deliver appropriate answers to users in real time in order to overcome some of the problems associated with the current online communication systems as stated in Chapter 1. Black-box approach has been used by other researchers in question-answering system whether in open domain (Nyberg \& Mitamura, 2002) or restricted domain (Diekema et al., 2004) as well as for conversational bots (Goh et al., 2007; Preez et al., 2009). Turing test which has high regard in this field is also a black-box approach (Floridi, 2003).

\subsection{Dataset}

Datasets are required to examine the responses generated by UniBot in black-box evaluation. For this purpose, one hundred questions are used.

\subsection{Dataset's results}

From the results shown in Table1, Uni-bot was accurately able to score 47 points out of 100 points. The scores are grouped according to the frequency so that trend can be observed in Table 5.5 and Figure 1. 
Table 1. Frequency of points

\begin{tabular}{|c|c|c|}
\hline Point(s) & Frequency & Percentage (\%) \\
\hline 4-Point & 47 & 47 \\
\hline 3-Point & 20 & 20 \\
\hline 2-Point & 12 & 12 \\
\hline 1-Point & 16 & 16 \\
\hline 0-Point & 5 & 5 \\
\hline Total & 100 & 100 \\
\hline
\end{tabular}

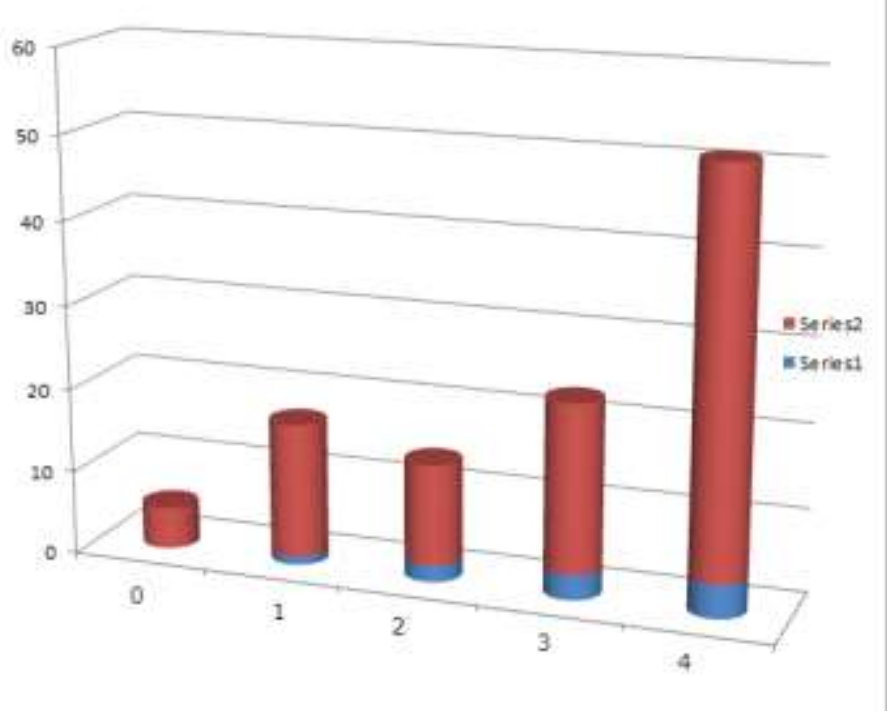

Figure 1. Scores frequency chart

From the table and chart shown in Table 1 and Figure 1 respectively, it can be seen that the majority of the dataset results fall at 4-point score. This means that Uni-bot was able to provide correct answers at most of the time during the test and some even with creative answers. Out of the hundred questions, 5 percent of the answers are in categories of o-point to 2-point. This shows that Uni-bot was able to response appropriately by at least giving some relevant answers. However, the existence of o-point results means that there are still some questions ended up with completely irrelevant responses. In this case, Uni-bot still needs to be further improved in the future.

\subsection{Summary}

This chapter saw the evaluation of UniBot being conducted using one hundred datasets compiled from online chat communication logs from KU Online Communication Department. The evaluation is done using the black-box approach which completely relied on the outputs generated and in this case are UniBot's responses. From the results gathered, it can be seen that UniBot was able to provide correct answers at most of the time and only small percentage of 5 percent having unrelated responses. 
I. Kuria \& H. Njoroge - Intelligent Conversational Agent for Enhancement of Online Communication ...

In terms of the objectives of this project, the evaluation showed that UniBot demonstrated the success of achieving the three objectives stated in Chapter 1.

\subsection{Conclusion}

This research has demonstrated a conversational agent for a university website users implemented using AIML. The evaluation results have shown that UniBot has great potential to interact with end users. Even though the knowledge in this study is limited to a single university, the solutions is customizable to fit any university setting. The advantages of UniBot such as being able to provide 24-hour service that can serve different time zones, able to have knowledge in multiple domains, and one conversational bot can be shared by multiple sites simultaneously have outweighed its existing limitations which are mainly due to external factors.

\section{Acknowledgements}

This research did not receive any specific grant from funding agencies in the public commercial, or not-for-profit sectors.

The authors declare no competing interests.

\section{References}

André, E., Rist, T., \& Müller, J. (1997). A life-like presentation agent for educational applications on the World-Wide Web. In Workshop on Intelligent Educational Systems on the World Wide Web at Artificial Intelligence in Education. Kobe, Japan.

Benifatemi, A. (2017). Preparing for an AI-driven society. Artificial Intelligence Emerging Trends.

Dan Ding, R., Cooper, A., \& Pearlman, J. (2007). Incorporating participatory action design into research and education. Retrieved from http://icee2007.dei.uc.pt/proceedings/papers/553.pdf.

Elis, R., Allen, T., \& Tuson, A. (2010). Applications and innovations in intelligent systems. Pg 180.

Graesser, A. C., Moreno, K., Martineau, J., Adcock, A., Olney, A., \& Person, N. (2003). Auto Tutor improves deep learning of computer literacy: Is it the dialogue or the talking head? In Artificial Intelligence in Education. Amsterdam: IOS Press.

Kerly, A., Hall, P., \& Bull, S. (2006). Bringing Chatbots into education: Towards natural language negotiation of open learner modelS, in R. Ellis, T. Allen \& A. Tuson (Eds.), Applications and innovations in intelligent systems XIV - Proceedings of AI-2006, 26 ${ }^{\text {th }}$ SGAI International Conference on Innovative Techniques and Applications of Artificial Intelligence, Springer.

Latham, A., Crockett, K., McLean, D., \& Edmonds, E. (2012). Adaptive tutoring in an intelligent conversational agent system.

Lester, J.C., Stone, B. A., \& Stelling, G. D. (1999). Lifelike pedagogical agents for mixed-initiative problem solving in constructivist learning environments. User modeling and user-adapted interaction. p. 1-44.

McCorduck, P. (2004). Machines who think. https://monoskop.org/images/1/1e/McCorduck Pamela Machines Who Think 2nd ed.p df. 
Perich, M. (2015). How students look for and choose their perfect education programmes online Retrieved from $\quad$ https://www.thestudyabroadportal.com/studyabroadblog/students-online-howstudents-look-for-and-choose-their-perfect-education-programmes-online/.

Person, N. K., \& Graesser, A. C. (2006). Pedagogical agents and tutors. In Encyclopedia of Education, J. W. Guthrie, Editor. Macmillan: New York, pp. 1169-1172.

Ser Ling Lim1, Ong Sing Goh (2016). Intelligent Conversational Bot for Massive Online Open Courses (MOOCs).

Sheth, R., (2003). Avatar technology: Giving a face to the e-learning interface. E-Learning Developer's Journal.

Shaw, E., Johnson, W. L., \& Ganeshan, R. (1999). Pedagogical agents on the Web, in International Conference on Autonomous Agents (pp. 283-290). ACM Press: Seattle, WA, USA.

The Society for the Study of Artificial Intelligence and Simulation of Behavior (2018). Retrieved from http://www.aisb.org.uk/events/loebner-prize.

Turing, A. M. (1950). Computing machinery and intelligence. Mind. LIX, (236), 433-460. http://doi.org/10.1093/mind/lix.236.433

Wallace, R. S. (2009). The anatomy of A.L.I.C.E. In R. Epstein, G. Roberts \& G. Beber (Eds.), Parsing the Turing Test. Springer, Dordrecht. https://doi.org/10.1007/978-1-4020-6710-5_13

Weizenbaum, J. (1966). ELIZA - A computer program for the study of natural language communication between man and machine. Communications of the ACM, 9(1), 36-45.

Apple Siri (2018). Retrieved from http://www.apple.com/siri/.

Microsoft Cortana (2018). Retrieved from https://www.microsoft.com/en-us/cortana.

Google Assistant (2018). Retrieved from https://assistant.google.com/\#?modal active=none.

Amazon Alexa (2018). Retrieved from https://developer.amazon.com/alexa. 
I. Kuria \& H. Njoroge - Intelligent Conversational Agent for Enhancement of Online Communication ...

C O A s 